\title{
Über einen bemerkenswerten Fall von Polysomatie in Wurzelspitzen
}

\author{
G. Reese ${ }^{1}$ \\ Botanisches Institut der Universität Kiel, Kiel, BRD
}

Eingegangen am Januar 27. 1972

Polysomatie, das gemeinsame Vorkommen von Zellen unterschiedlicher Ploidiestufe im gleichen Gewebe oder Individuum, scheint für die Angiospermen typisch zu sein und ist nicht selten auch in ihren Wurzelspitzen nachweisbar. Nach den ersten Hinweisen zu Beginn des Jahrhunderts sind vor allem in den letzten drei Jahrzehnten zahlreiche Einzelbeobachtungen an den verschiedensten Taxa hinzugekommen und auch in Sammelreferaten mehrfach zusammengestellt und kritisch gesichtet worden (vgl. Tischler u. Wulf 1953-63). Die meisten Fälle gehen auf Endopolyploidisierungsprozesse beim Übergang vom Teilungs- zum Streckungswachstum zurück. Treten die derart polyploid gewordenen Zellen nochmals in Mitosen ein oder heben sich durch einen andersartigen Formwechsel ihrer Chromozentren und ein größeres Kernvolumen von den diploid gebliebenen ab, so fällt der Nachweis der Polysomatie nicht schwer. Der Cytochimärencharakter der Sproß- oder Wurzelspitzen wird unter den geschilderten Verhältnissen im Übergangsbereich zwischen der meristematischen und der Streckungszone sichtbar. Sollte ein solcher Nachweis über Mitosen polyploider Kerne möglich sein, so lassen die nicht selten paarweise oder auch $\mathrm{zu}$ viert angeordneten Chromosomen mit Sicherheit auf kürzlich voraufgegangene Endomitosen schließen.

Aber nicht alle polysomatischen Wurzelspitzen lassen sich einem solchen Regelfall zuordnen. Bei den in den letzten Jahren auf ihre Chromosomenverhältnisse untersuchten Stapelieen (Reese u. Kressel 1967, Reese 1971) erfordert die hier keineswegs seltene Polysomatie offenbar eine andere, ungewöhnlichere Deutung.

\section{Material und Methode}

Die Untersuchungen erfolgten an Nawaschin-fixierten und zu $10 \mu$ dicken, Hämatoxylin-gefärbten Mikrotomschnitt-Dauerpräparaten verarbeiteten Wurzelspitzen verschiedener in den Gewächshäusern des Botanischen Gartens Kiel kultivierter Stapelieen (Asclepiadaceae); darunter sowohl taxonomisch exakt definierbare Species von Wildfundorten wie auch unsichere Taxa nicht bekannter Herkunft.

\section{Ergebnisse}

Die Besonderheit mixoploider Stapelieen-Wurzeln besteht, zunächst rein deskriptiv, darin, daß die Zellen mit der abweichenden, in der Regal um das Doppelte erhöhten Chromosomenzahl nicht erst in einiger Entfernung vom Vegetations-

$1+24$. Juli 1972 . 
punkt registriert werden-was als Endopolyploidisierungsfolge im Zuge der Differenzierung verständlich wäre, sondern bereits im Spitzenmeristem des Vegetationsskegels nachweisbar sind.

Das Ausmaß der Polysomatie läßt dabei verschiedene Abstufungen erkennen:

1) Die verdoppelte Chromosomenzahl tritt, beurteilt nach Querschnitten nur in Einzelzellen oder wenigen auf.

Dieser Fall ist relativ häufig; Beispiele anzuführen erübrigt sich.

2) Die verdoppelte Chromosomenzahl tritt in größeren Zellkomplexen, oder Gewebeschichten auf; es können so Organe von sektorialem, peri- oder meriklinalem Cytochimärencharakter entstehen.

Beispiele (außer den nachstehend beschriebenen polysomatischen Wurzelspitzen wurden bei allen genannten Arten auch monosomatische mit der artspezifischen Chromosomenzahl beobachtet):

a) Stapelia schinzii Bgr. et Schltr. ( $2 \mathrm{n}=22$; Kiel-Nr. 260)

Kurzer meriklinaler, tetraploider Abschnitt aus Dermatogen- und wenigen Periblemzellen

b) Decabelone elegans Decne. $(2 \mathrm{n}=22$; Kiel-Nr. 336) und

Huernia loeseneriana Schltr. $(2 \mathrm{n}=22$; Kiel-Nr. 363)

Bis ans Plerom reichender sektorialer Abschnitt von etwa $90^{\circ}$ aus tetraploidem Dermatogen und Periblem

c) Stapelia spec. (erhalten als $S$. nobilis N.E.Br.) $(2 \mathrm{n}=22$; Kiel-Nr. 237) Bis ins Plerom reichender Sektor von etwa $140^{\circ}$ aus tetraploiden Zellen

d) Huerniopsis atrosanguinea (N.E. Br.) White et Sloane ( $2 n=22 ;$ Kiel-Nr. 130) Unter zahlreichen diploiden Wurzelspitzen mit $2 n=22$ eine tetraploide $(2 n=44)$ mit oktoploidem Dermatogen $(2 n=88)$ und vermutlich auch oktoploider Kalyptra

e) Stapelia spec. (erhalten als S. wilmaniae Luckh.) $2 \mathrm{n}=22 ; \mathrm{Kiel}-\mathrm{Nr}$. 9) Periklinalchimäre mit diploidem Dermatogen und Periblem und tetraploidem Plerom

f) Piaranthus spec. aff. ruschii $\mathrm{Nel}(2 \mathrm{n}=22$; Kiel-Nr. 191)

Tetraploide Wurzelspitze mit einem peripher gelegenen, kleinen, kappenartigen Abschnitt aus diploiden Zellen.

Die beiden Kernvalenzstufen werden schon ohne Chromosomenzählungen aus den Zellgrößen deutlich (Abb. 1)

3) Die verschiedenen Ploidiestufen treten nicht innerhalb einer Wurzelspitze auf, sondern verteilen sich auf verschiedene Wurzelspitzen der gleichen Pflanze. Sie hat in diesem Falle teils diploide, teils tetraploide Wurzeln.

Beispiele:

Caralluma adenensis (Defl.) Bgr. ( $2 \mathrm{n}=22$; Kiel-Nr. 367) und Huernia brevirostris N.E. Br. $(2 \mathrm{n}=22$; Kiel-Nr. 332): 2 Wurzelspitzen mit $2 \mathrm{n}=22$, 1 mit $2 \mathrm{n}=44$; Stapelia spec. (erhalten als $S$. $\times$ bella Bgr.) $(2 \mathrm{n}=22$; Kiel-Nr. 45): 1 Wurzelspitze mit $2 \mathrm{n}=22,3$ mit $2 \mathrm{n}=44$; Stapelia spec. (aus der Gigantea-hirsuta-Gruppe) $(2 \mathrm{n}=22$; Kiel-Nr. 13): 6 Wurzelspitzen mit $2 \mathrm{n}=22,1$ mit $2 \mathrm{n}=44$; Stapelia peculiaris Luckh. $(2 n=22$; Kiel-Nr. 401): 1 Wurzelspitze mit $2 n=22,2$ mit $2 n=44$. 


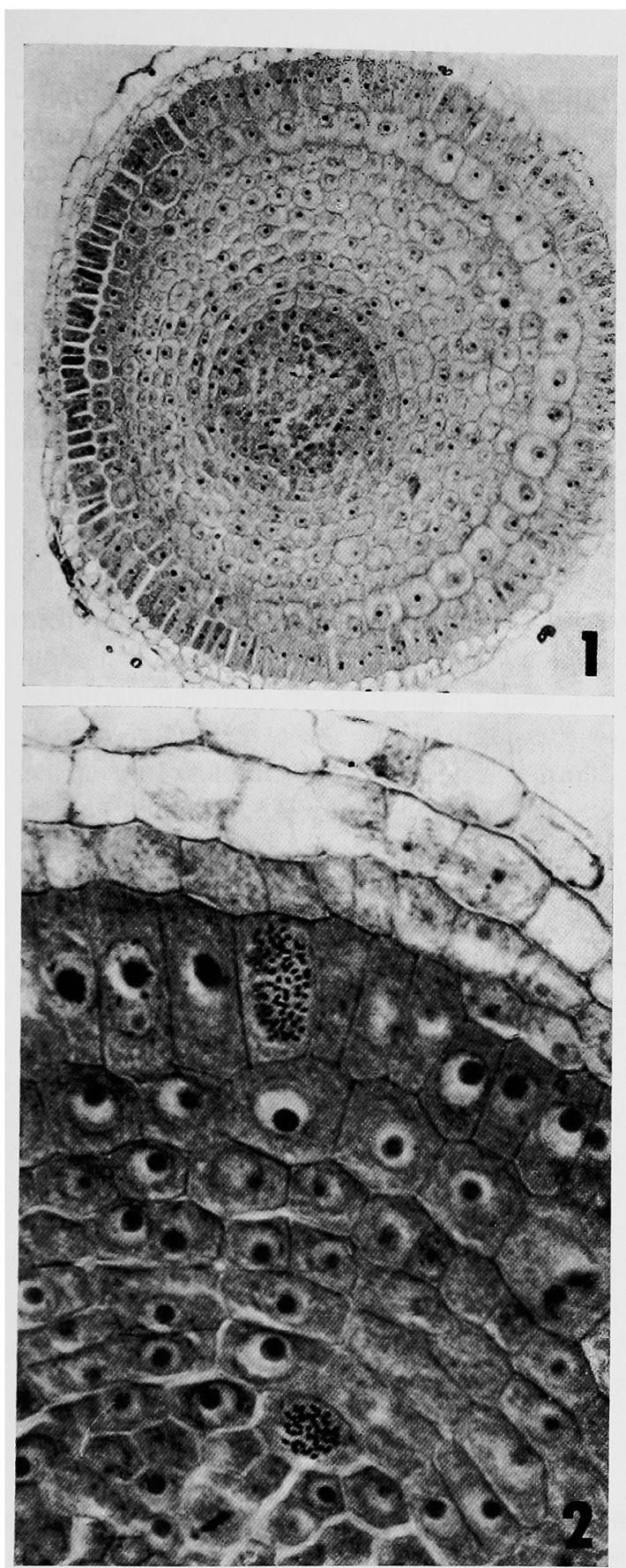

Abb. 1. Tetraploide Wurzelspitze mit diploidem peripherem Abschnitt (links im Bild); Piaranthus spec. aff. ruschii. Vergr. $190 \times$

Abb. 2. Metaphase im Dermatogen mit $2 n=88$, im Plerom mit $2 \mathrm{n}=44$; Duvalia reclinata. Vergr. $700 \times$
Die Übergänge zwischen den unter 1) bis 3) aufgeführten Abstufungen sind naturgemäß fließend.

Außer diesen im Hinblick auf die Verdoppelung oder auch Vervierfachung noch als "regulär" anzusehenden und kausal verständlichen Fällen von Polysomatie seien noch zwei weitere erwähnt, bei denen die chromosomal aberranten Zellen nicht durch einfache Duplikationsvorgänge erklärt werden können.

a) Bei der bereits unter den Beispielen zu 3) genannten Stapelia spec. aus der Giganteahirsuta-Gruppe wurde inmitten eines diploiden Wurzelspitzenmeristems $(2 n=22)$ eine einzelne Metaphaseplatte mit $2 n=64-66$ beobachtet.

b) Der zweite Fall bezieht sich auf Duvalia reclinata (Mass.) Haw. (2n=44; Kiel-Nr. 82), eine tetraploide Pflanze, die auch unter den oben genannten Beispielen für Polysomatie stehen könnte. Denn bei ihr erwiesen sich alle drei untersuchten Wurzelspitzen als mixoploid im herkömmlichen Sinne. Außer den tetraploiden Mitosen wurden relativ häufig im Dermatogen und Periblem auch oktoploide gefunden (Abb. 2). Diese Zellen mit $2 \mathrm{n}=88$ sind $\mathrm{zwar}$ nicht im Sinne charakteristischer Chimärenmuster angeordnet, doch kommen neben oktoploiden, im Querschnitt als Einzelzellen nachweisbare Zellreihen auch mehrzellige oktoploide Komplexe vor. Sie liegen überwiegend im Dermatogen. Auch die Kalyptra scheint zumindest 
partiell hochpolyploid zu sein.

Die Besonderheit dieser Duvalia liegt jedoch nicht in den tetra-/oktoploiden Mischgeweben, sondern darin, daß außerdem noch-mit Sicherheit wenigstens bei einer Metaphase im Dermatogen-2n=ca. 132 gezählt werden konnten.

In beiden Fällen besteht die Irregularität in einer Verdreifachung der Ausgangschromosomenzahl.

Bevor versucht wird, die ungewöhnliche Polysomatie der Stapelieen-Wurzeln (von den beiden zuletzt genannten Sonderfällen sei vorerst abgesehen; vgl. hierzu S 599) kausal zu deuten, muß ausdrücklich betont werden, daß in den Teilungsstadien der Kerne mit erhöhten Ploidiestufen niemals eine Paarbildung der Chromosomen beobachtet wurde, auch nicht im Meristem des Vegetationskegels.

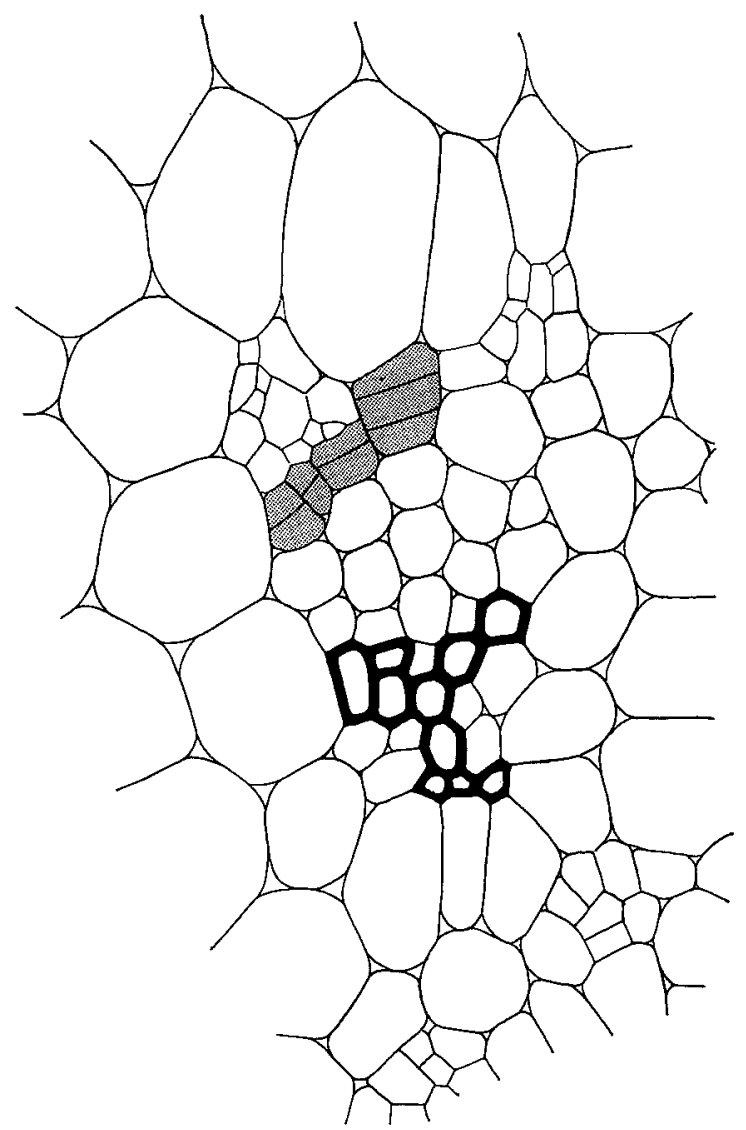

Abb. 3. Bikollaterales Leitbündel aus dem Achsengewebe mit beginnender Differenzierung eines Wurzelprimordiums (Initialen dunkel getönt); Duvalia reclinata. Vergr. $35 \times$

Damit kann man zur Erklärung der Polysomatie-vor allem im Hinblick darauf, $\mathrm{da} B$ sie in den vorstehend geschilderten Fällen nicht erst in einiger Entfernung vom Vegetationspunkt, sondern auch schon in seinen Initialen auftritt-meines Erachtens nur annehmen, daß an der Bildung der Wurzelprimordien a priori Zellen unter- 
schiedlicher Kernvalenz beteiligt sind oder sein können; also neben den mit der artspezifischen Chromosomenzahl ausgestatteten auch solche, die im Zuge der Ausdifferenzierung in ihrem "Mutter"-Organ über Endomitosen bereits eine Verdoppelung der Chromosomenzahl erfahren haben.

Diese Vorstellung wird auch dadurch gestützt, daß die bei den Stapelieen für Chromosomenuntersuchungen fixierten Wurzeln häufig sproßbürtige Adventivwurzeln sind. Die stammsukkulenten Stapelieen werden ganz überwiegend durch Stecklinge vermehrt und haben vielfach auch kriechende Achsen. Die aus ihnen hervorbrechenden Adventivwurzeln entstehen endogen aus den Bereichen des Außenphloems (Abb. 3) und sind mit ihren Primordien nicht an eine klar abgrenzbare Zellschicht gebunden, wie das bei den Seitenwurzeln mit dem Perizykel der Fall $\mathrm{zu}$ sein pflegt. Ich halte es für wahrscheinlich, daß vor allem die Adventivwurzeln selbst zur Polysomatie im apikalen Meristem neigen, weniger dagegen ihre im Zuge der cytologischen Bearbeitung ebenfalls mit erfassten Seitenwurzeln. Diese Annahme ist allerdings unbewiesen, weil zu Beginn der Untersuchungen nicht auf eine Trennung der beiden Wurzeltypen geachtet wurde. Doch ist generell wohl nicht $\mathrm{zu}$ bezweifeln, daß die Entstehung der mixoploiden Stapelieen-Wurzelspitzen nur über die Beteiligung mehrerer Zellen an den endogenen Wurzelanlagen verständlich wird, wobei unterstellt werden muß, daß auch Dauergewebszellen mit endomitotisch erhöhter Chromosomenzahl sekundär meristematisch werden und an der Wurzelbildung ohne nachweisbare Beeinträchtigung der Pflanze mitwirken können.

Der endgültige Beweis für die Richtigkeit dieser Vorstellungen kann nur durch die unmittelbare Beobachtung der cytologischen Vorgänge bei der Primordienbildung erfolgen. Er dürfte selbst bei einem enormen Materialaufwand nur mit viel Glück zu führen sein. Unter der gegebenen Zielsetzung aber ist es schon ein Fortschritt, wenn sich in der parenchymatischen Region des Außenphloems der sukkulenten Sprosse endopolyploidisierte Zellen nachweisen lassen. Das ist auf Grund der Größe, Struktur und des Formwechsels von Endochromozentren und an Hand der Kerngrößen und induzierter Mitosen für die Stapelieen auch bereits durch Czeika (1956) geschehen; allerdings nur für Echidnopsis cereiformis, ein in der vorliegenden Arbeit nicht berücksichtigtes Taxon. Er wies unter anderem nach, daß das Rindengewebe bei dieser Species durchschnittlich 16-ploid, das Markgewebe vermutlich 32-ploid wird. Außerdem fand er "eigenartige im Phloem der Gefäßbündel liegende parenchymatische, in der Jugend plasmareiche Zellen”, die, zunächst einkernig und 8- bis 16-ploid, später vermutlich durch Kernfragmentationen vielkernig werden.

\section{Besprechung der Ergebnisse}

Schon bei früheren cytologischen Untersuchungen an Stapelieen waren die relativ häufig zu beobachtenden Teilungen mit verdoppelten Chromosomenzahlen aufgefallen und auch von Reese u. Kressel (1967) mit dem Bemerken, daß es sich um "postendomitotische Metaphasen" handle, ausdrücklich erwähnt worden. Das ist selbstverständlich nur im weitesten Sinne zu verstehen, denn die die Polysomatie 
der Apikalmeristeme bedingenden Endomitosen erfolgen ja nicht in den Wurzeln selbst, sondern in den "Mutter"-Organen, in denen ihre Primordien angelegt werden.

Die im Hauptteil dieser Arbeit entwickelte kausale Deutung der Polysomatie in den Wurzelspitzen der Stapelieen steht im Widerspruch zu der herkömmlichen Vorstellung. Diese hat zweifellos für die Mehrzahl der einschlägigen Befunde durchaus ihre Berechtigung. Sie basiert, wie einleitend bereits kurz umrissen, auf der Beobachtung, daß im Anschluß an die Teilungsphase der über ein Apikalmeristem wachsenden Organe ein Teil der Zellen in diesem Organ selbst endopolyploidisiert wird. Die Mixoploidie tritt dann frühestens im Grenzberich zwischen der Teilungs- und Streckungszone auf.

Es gibt jedoch, bezogen auf die Lage der Endomitosen im Laufe der Ontogenese, noch einen weiteren Modus. Er wurde zuerst für Spinacia oleracea von Berger (1941) nachgewiesen. Hier findet die endomitotische Polyploidisierung bereits im Embryo vor Abschluß der Samenreife statt; die Keimlingswurzeln haben daher bei dieser Art ein von vornherein polyploides Periblem. Gleiches gilt im Prinzip auch für andere Angiospermen.

Die dritte Ableitung der Polysomatie, wie sie in der vorliegenden Arbeit für die Stapelieen wahrscheinlich gemacht werden konnte, ist bisher wenig beachtet worden; zumindest wurde ihre Eigenart gegenüber den anderen Modi nicht gebührend herausgestellt. Die ersten Untersuchungen, in denen ausdrücklich auf die gleiche oder eine ähnliche Begründung für die Mixoploidie wie bei den Stapelieen geschlossen wird, scheinen die von Levan (1944) an Beta vulgaris zu sein. Er fand bei der cytologischen Überprüfung der "second-year root tips", also solchen, die nach Überwinterung der geernteten Rüben im zweiten Jahr neu angelegt werden, bei etwa $10 \%$ der Pflanzen auf das Doppelte erhöhte Chromosomenzahlen, die sich entweder auf einzelne Sektoren oder auch die ganze Wurzel bezogen. Im letzteren Falle entwickelte die betreffende Rübe somit zwei cytologisch differente, aber in sich einheitliche Wurzeltypen: neben den mit der sorteneigenen Chromosomenzahl auch solche mit einer um das Doppelte erhöhten. Vergleiche mit Zuckerrübenkeimlingen ergaben dagegen nur einen Anteil von $0.2 \%$ mixoploider Pflanzen. Zur Begründung der hochgradigen Polysomatie der "second-year root tips" schreibt Levan: "It is easily understandable that the secondary root meristems of the old beets may contain cells which during their development have undergone endomitosis."

D'Amato (1952) nennt noch zwei weitere Arbeiten, aus deren Ergebnissen ebenfalls auf die Bildung von Wurzelanlagen aus endopolyploidisierten, sekundär ihre Teilungsfähigkeit wiedererlangenden Dauergewebszellen geschlossen werden kann: a) eine eigene Veröffentlichung aus dem Jahre 1950; 2 von 43 Wurzeln einer diploiden Zwiebel von Allium cepa erwiesen sich als hochgradig mixopolyploid mit Kernvalenzen zwischen $4 \mathrm{n}$ und vermutlich $32 \mathrm{n}$; er glaubt allerdings, daß ein Bakterienbefall oder eine zufällige Verwundung diese Anomalie hervorgerufen haben müsse; b) eine Arbeit von Gaiser (1949) über die Compositen-Gattung Liatris; er fand in zwei Fällen unter den aus überwinterten Rhizomknollen hervorgehenden Wurzeln statt der erwarteten Diploidzahlen Tetra- und Hexaploidie.

In den meisten Fällen sind letztlich Endomitosen, wann auch immer im Ver- 
laufe der Ontogenese, die Ursache für die Polysomatie. Das kann sensu stricto jedoch nur dann gelten, wenn die Ausgangschromosomenzahl im Sinne einer Verdoppelungsreihe vervielfacht wird. In anderen Fällen aber, wie beispielsweise Triploidie, Hexaploidie und Aneuploidie (vgl. die beiden auf S... beschriebenen Beobachtungen), bleibt man auf Spekulationen angewiesen und wird in der Regel Mitose- oder auch Endomitose-Anomalien zur Erklärung heranziehen. So ließe sich vielleicht auch ein Zusammenhang mit den Kernfragmentationen sehen, die Czeika (1956) für einige endopolyploidisierte Kerne im Phloem der Gefäßbündel von Echidnopsis (Stapelieae) wahrscheinlich machen konnte (vgl. auch S 596). Der mikroskopische Nachweis für Endomitose-Anomalien steht aber wohl noch aus, denn man kennt zwar die verschiedensten Erscheinungsformen der Endomitose (vgl. das erst kürzlich erschienene Sammelreferat von Tschermak-Woess 1971), auch zahlreiche Übergänge zu echten Mitosen, aber daß etwa von der endomitotischen Verdoppelung nur eines der beiden Genome oder nur wenige oder Einzelchromosomen betroffen werden können, sind meines Wissens bislang unbewiesene Modellvorstellungen. Tschermak-Woess ist im übrigen der Ansicht, daß die Endomitose wegen Fehlens der Kernspindel weniger störanfällig sei als die Mitose.

Bei den Stapelieen kommen anscheinend häufiger als bei anderen Pflanzengruppen, vielleicht wegen der relativ zahlreichen sproßbürtigen Adventivwurzeln, Exemplare vor, die außer diploiden auch rein tetraploide Wurzeln besitzen. Diese Feststellung muß sich zwangsläufig auch auf die Beurteilung von Chromosomenzählungen für die Stapelieen-Arten auswirken. Kann man überhaupt sicher sein, daß der ermittelte Diploidwert tatsächlich der Chromosomenzahl des untersuchten Taxons entspricht, oder hat man zufällig Teilungen mit verdoppelter Chromosomenzahl ausgewertet? Eine zweifellos berechtigte Frage, die sich vor allem der Verf. selbst vorzulegen hat, der in den vergangenen Jahren (Reese u. Kressel 1967, Reese 1971) für rund 110 Arten die somatischen Chromosomenzahlen veröffentlicht hat.

Mir schiene es verfehlt, der Polysomatie im Zusammenhang mit der offenkundigen Euploidie innerhalb der Stapelieen allzu große Bedeutung beizumessen. Zwar ist nicht zu verkennen, daß sie den flüchtigen Beobachter zu Fehlinterpretationen verleiten kann, doch wird man nicht sagen können, daß die Entdeckung der polyploiden Arten wesentlich der zufälligen Untersuchung ausnahmsweise polyoder mixoploider Wurzeln zuzuschreiben sei. Wie Überprüfungen von mehreren Exemplaren eines Taxons an mehreren Wurzelspitzen und in verschiedenen Jahren zeigen, hat der größte Teil der Pflanzen eine taxonspezifisch konstante Chromosomenzahl. Trotzdem bleibt Vorsicht am Platze, denn völlig ausschließen lāßt sich eine Fehlbeurteilung letztlich nur über einen erheblichen, vielfach gar nicht durchführbaren Materialaufwand.

Hinzuweisen wäre noch auf die Bedeutung, die man der Produktion von Organen mit abweichenden Chromosomenzahlen für die Entstehung neuer Cytotypen auf vegetativem Wege beimessen kann. Denn was für Wurzelprimordien gilt, mag auch für Seitensproßanlagen zutreffen. Sharma hat schon 1956 nachdrüchlich auf diese Möglichkeiten hingewiesen, ohne allerdings einen experimentellen Nachweis führen zu können. 


\section{Zusammenfassung}

1. In den Wurzelspitzen (vermutlich überwiegend sproßbürtigen Adventivwurzeln) der stammsukkulenten Stapelieen (Asclepiadaceae) tritt relativ häufig Polysomatie auf. Ihr Ausmaß, beurteilt nach Querschnitten, läßt verschiedene Abstufungen erkennen:

a) Die Zellen mit verdoppelter Chromosomenzahl finden sich einzeln oder zu wenigen;

b) größere Komplexe von ihnen verleihen den Wurzeln den Charakter von sektorialen, meri- oder periklinalen Cytochimären.

c) Verschiedene Wurzeln einer Pflanze haben entweder die taxonspezifische Chromosomenzahl oder eine um das Doppelte erhöhte; in diesem Falle ist nicht die Wurzel polysomatisch, sondern die Pflanze.

2. Die Polysomatie ist bereits in den Initialen des Apikalmeristems nachweisbar, macht sich also nicht erst beim Übergang von der Teilung zur Streckungszone bemerkbar. In den Teilungsphasen der Zellen mit verdoppelter Chromosomenzahl wurden niemals gepaart liegende Chromosomen beobachtet.

3. Entgegen der üblichen Deutung, daß die Polysomatie in der Regel als Folge einer Endopolyploidisierung im Zuge der Zelldifferenzierung in dem betroffenen Organ selbst zu verstehen ist, muß für die Stapelieen angenommen werden, daß sich bereits an der Bildung der Adventivwurzelprimordien mehrere Zellen unterschiedlicher Kernvalenz beteiligen, die teilweise schon im "Mutter"-Organ Endomitosen durchgemacht haben.

Die Primordien werden in der Region des Außenphloems angelegt.

4. Außer der "regulären" Polysomatie werden zwei Fälle beschrieben, in denen die erhöhte Chromosomenzahl einer Verdreifachung des Ausgangswertes entspricht.

\section{Summary}

On a remarkable case of polysomaty in root tips

1. Polysomaty can be observed relatively often in root tips (probably of adventitious roots) in the Stapelieae (Asclepiadaceae). Its degree, estimated by crosssections, allows assessment of different graduations:

a. The cells with the double number of chromosomes can be observed as solitary or in small groups.

b. Greater complexes of these cells give the roots the character of sectorial, meri- or periclinal cytochimaeras.

c. Different roots of the same plant either show the taxonspecific number of chromosomes or doubled one. In this case it is not the root that is polysomatic, but the plant.

2. Polysomaty is already observable in the initials of the apical meristem. Chromosomes grouped in pairs have never been observed in cells containing double number of chromosomes.

3. Thus it must be concluded that, in the Stapelieae, several cells with a different 
level of ploidy participate in the formation of the root primordias. Partly these cells apparently have undergone endomitosis in their "mother"-organ. This is not in accordance to the usual view that polysomaty is due to the endopolyploidization in the course of cell differentiation, which occurs in the concerning organ before starting cell elongation.

The primordias of the adventitious roots are being produced in the region of the external phloem.

\section{Literatur}

d'Amato, F. 1950. Occasionale comparsa di radici altamente poliploidi in un bulbo di cipolla. Caryologia 2: 160-164.

- 1952. Polyploidy in the differentiation and function of tissues and cells in plants. Caryologia 4: 311-358.

Berger, C. A. 1941. A reinvestigation of polysomaty in Spinacia. Bot. Gaz. 102: 759-769.

Czeika, G. 1956. Strukturveränderungen endopolyploider Ruhekerne im Zusammenhang mit wechselnder Bündelung der Tochterchromosomen und karyologisch-anatomische Untersuchungen an Sukkulenten. Österr. Bot. Z. 103: 536-566.

Gaiser, L. O. 1949. Chromosome studies in Liatris. I. Spicatae and Pycnostachyae. Am. J. Bot. 36: 122-135.

Levan, A. 1944. On the normal occurrence of chromosome doublings in second-year root tips of sugar beets. Hereditas 30: 161-164.

Reese, G. 1971. Untersuchungen über die Chromosomenzahlen der Stapelieae II. Portug. Acta Biol., Ser. A, 12: 1-23.

- u. Kressel, H. 1967. Untersuchungen über die Chromosomenzahlen der Stapelieae. Portug. Acta Biol., Ser. A 10: 33-54.

Sharma, A. K. 1956. A new concept of a means of speciation in plants. Caryologia 9: 93-130.

Tischler, G., u. Wulff, H. D. 1953-1963. Allgemeine Pflanzenkaryologie, Erg.-Bd. "Angewandte Pflanzenkaryologie". In Handb. d. Pflanzenanatomie, Bd. 2, Berlin.

Tschermak-Woess, E. 1971. Endomitose. In Handb. d. Allg. Pathol., Bd. 2, T1. 2, Berlin-Heidelberg-New York. 